Article

\title{
Plasmon-Enhanced Blue-Light Emission of Stable Perovskite Quantum Dot Membranes
}

\author{
Kai Gu ${ }^{1}$, Hongshang Peng ${ }^{1,2}$, Siwei Hua ${ }^{1}$, Yusong $Q u^{1}$ and Di Yang ${ }^{1, *}$ \\ 1 College of Science, Minzu University of China, Beijing 10081, China; kai_gu94@163.com (K.G.); \\ hshpeng@bjtu.edu.cn (H.P.); 17301037@muc.edu.cn (S.H.); quyusong123@126.com (Y.Q.) \\ 2 Key Laboratory of Luminescence and Optical Information, Ministry of Education, \\ Institute of Optoelectronic Technology, Beijing Jiaotong University, Beijing 100044, China \\ * Correspondence: diyang@muc.edu.cn; Tel.: +86-1352-274-3208
}

Received: 18 April 2019; Accepted: 15 May 2019; Published: 19 May 2019

check for updates

\begin{abstract}
A series of stable and color-tunable $\mathrm{MAPbBr}_{3-x} \mathrm{Cl}_{x}$ quantum dot membranes were fabricated via a cost-efficient high-throughput technology. $\mathrm{MAPbBr}_{3-\mathrm{x}} \mathrm{Cl}_{\mathrm{x}}$ quantum dots grown in-situ in polyvinylidene fluoride electrospun nanofibers exhibit extraordinary stability. As polyvinylidene fluoride can prevent the molecular group $\mathrm{MA}^{+}$from aggregating, $\mathrm{MAPbBr}_{3-\mathrm{x}} \mathrm{Cl}_{\mathrm{x}}$ quantum dots are several nanometers and monodisperse in polyvinylidene fluoride fiber. As-prepared $\mathrm{MAPbBr}_{3-x} \mathrm{Cl}_{x}$ quantum dot membranes exhibit the variable luminous color by controlling the $\mathrm{Cl}^{-}$content of $\mathrm{MAPbBr}_{3-\mathrm{x}} \mathrm{Cl}_{\mathrm{x}}$ quantum dots. To improve blue-light emission efficiency, we successfully introduced $\mathrm{Ag}$ nanoparticle nanofibers into $\mathrm{MAPbBr}_{1.2} \mathrm{Cl}_{1.8}$ quantum dot membranes via layer-by-layer electrospinning and obtained $\sim 4.8$ folds fluorescence enhancement for one unit. Furthermore, the originality explanation for the fluorescence enhancement of $\mathrm{MAPbBr}_{3-\mathrm{x}} \mathrm{Cl}_{\mathrm{x}}$ quantum dots is proposed based on simulating optical field distribution of the research system.
\end{abstract}

Keywords: perovskite quantum dots; plasmon-enhance fluorescence; electrospinning

\section{Introduction}

Hybrid organic-inorganic perovskites have made remarkable achievements in the application of photovoltaic devices [1], but beyond that they have garnered considerable attention in light-emitting applications due to their low-cost, facile preparations [2], flexible tunability of emission light [3], extraordinary charge-transport properties [4], and promisingly ultrahigh photoluminescence $[5,6]$. However, hybrid organic-inorganic perovskites suffer from environmental instability and are frustrated in light-emitting applications [7]. As an important class of hybrid organic-inorganic perovskites, $\operatorname{MAPbX}_{3}\left(\mathrm{MA}=\mathrm{CH}_{3} \mathrm{NH}_{3}{ }^{+}, \mathrm{X}=\mathrm{Cl}, \mathrm{Br}\right.$, I) has been widely believed to possess an inoxidizability ground state in oxygen atmospheres, and more importantly, when $\mathrm{MAPbX}_{3}$ quantum dots (QDs) are exposed to oxygen atmospheres, their photoluminescence (PL) intensities are enhanced [8]. Recently, $\mathrm{MAPbX}_{3}$ QDs has become a promising candidate in breaking through the short-term stability and gain important positions in many light-emitting applications such as light-emitting diodes (LEDs), lasers and light-emitting transistors $[9,10]$.

Among the family of $\mathrm{MAPbX}_{3}$ QDs, many reports have mainly focused on $\mathrm{MAPbBr}_{3}$ QDs, which is a green emitter with excellent photoluminescence quantum yield (PLQY, 90\%) [11,12]. By doping $\mathrm{MAPbBr}_{3}$ with chlorine, stoichiometric $\mathrm{MAPbBr}_{3-\mathrm{x}} \mathrm{Cl}_{\mathrm{x}}$ can be synthesized. The bandgap of $\mathrm{MAPbBr}_{3-\mathrm{x}} \mathrm{Cl}_{\mathrm{x}}$ widens as $\mathrm{Cl}^{-}$proportion increases and the emission wavelength can be tuned continuously from 400 to $520 \mathrm{~nm}$ by adjusting the ratio of $\mathrm{Cl}^{-}$to $\mathrm{Br}^{-}$of $\mathrm{MAPbBr}_{3-\mathrm{x}} \mathrm{Cl}_{\mathrm{x}}$ [13]. Unfortunately, with the increase of bandgap width of $\mathrm{MAPbBr}_{3-x} \mathrm{Cl}_{x}$, more defect energy levels located in the bandgap are likely to serve as deep level recombination centers, and $\mathrm{MAPbBr}_{3-x} \mathrm{Cl}_{x} \mathrm{QDs}$ 
generally suffer from low PLQY [14]. One major source of such defects is associated with dangling bonds on the surface of $\mathrm{MAPbBr}_{3-\mathrm{x}} \mathrm{Cl}_{\mathrm{x}}$ QDs and surface passivation is an effective approach to remove these and achieve efficient photoluminescence. The polymer matrices with compact molecular chains were reported to be effective in passivating perovskite quantum dots (PQDs) and the polymer-encapsulated PQDs exhibit high environment stability especially water resistance [15].

A cost-efficient high-throughput strategy for fabricating polymer-encapsulated PQDs has recently become one research hotspot due to its necessity and importance to the future industrialization of these materials. Spin-coating is usually used to fabricate large-area membranes. However, in a spin-coating process, there is not only a serious waste of materials, but it is also difficult to control the uniformity of large-area membranes [16]. These daunting problems disappear when electrospinning is used to prepare large-area membranes. More interestingly, electrospinning is quite capable of fabricating various functional nanofibers, thus leading to plentiful and novel performances from the large-area membranes made of the nanofibers. Recently, polymer-encapsulated PQDs prepared by electrospinning have attracted great attention [17-19]. For instance, Tsai et al. [20] used polyacrylonitrile and perovskite precursor as shell and core materials, respectively, and prepared core-shell coaxial nanofibers through one-step coaxial electrospinning method. They revealed that these nanofibers were endowed with high ambient stability and a high resistance to water. Kuo et al. [21] reported a novel electrospinning strategy for incorporating $\mathrm{CsPbX}_{3}(\mathrm{X}=\mathrm{Cl}, \mathrm{Br}, \mathrm{I})$ QDs into poly (styrene-butadiene-styrene). The protected PQDs continued to emit bright fluorescence for over $1 \mathrm{~h}$ when immersed in water.

Even more remarkably, electrospinning could knit nanofibers embodying plentiful plasmonic nanoparticles and PQDs together in the most optimal way, which ensures extraordinary fluorescence enhancement. Although plasmon-enhanced fluorescence has been widely employed to boost the luminescence yields of fluorescence dyes and QDs [22-26], few studies have focused on the fluorescence enhancement system that plasmonic nanofibers are interwoven with QD nanofibers. Therefore, there is a lack of thorough understanding of fluorescence enhancement and the interaction mechanism between the two types of nanofibers for this system, which is different from the traditional interactions between particles and/or films.

Herein, we successfully prepared uniform membranes of polymer-encapsulated $\mathrm{MAPbBr}_{3-\mathrm{x}} \mathrm{Cl}_{\mathrm{x}}$ QDs via a straightforward uniaxial electrospinning method. Polyvinylidene fluoride (PVDF) was selected to endow PQDs high stability due to its hydrophobic characteristics, semi-crystalline and strong ability to restrict PQDs growth [27]. The as-fabricated PVDF-encapsulated PQDs (PVDF-PQDs) membranes exhibit color-tunability by controlling the ratio of $\mathrm{Cl}^{-}$to $\mathrm{Br}^{-}$in $\mathrm{MAPbBr}_{3-\mathrm{x}} \mathrm{Cl}_{\mathrm{x}}$. On this basis, the $\mathrm{MAPbBr}_{3-\mathrm{x}} \mathrm{Cl}_{\mathrm{x}}$, which the PL spectrum coincides well with plasmonic resonance band of silver nanoparticles (NPs), were selected for Ag enhanced fluorescence experiments. Ag nanofibers were introduced into PQD nanofiber membrane via layer-by-layer electrospinning method and Ag nanofibers of each layer interweaved with PQD nanofibers of the adjacent layer. We defined PQDs-layer/Ag NPs-layer/ PQDs-layer as an analytical periodic unit and proved that one unit possesses a $\sim 4.8$ folds fluorescence enhancement, which is a significant improvement compared with $\sim 1.7$ folds fluorescence enhancement for $\mathrm{CsPbr}_{3}$ QDs and $\sim 4.0$ folds fluorescence enhancement for $\mathrm{MAPbBr}_{3}$ QDs reported elsewhere $[23,26]$. Furthermore, we interpreted the plasmonic enhancement mechanism of this system by simulating the light field distribution of Ag nanofibers.

\section{Materials and Methods}

\subsection{Materials}

$\mathrm{PbCl}_{2}$ (99.99\%), $\mathrm{MABr}\left(\mathrm{MA}=\mathrm{CH}_{3} \mathrm{NH}_{3}{ }^{+}, 99.5 \%\right), \mathrm{PbBr}_{2}(99.99 \%)$ and $\mathrm{MACl}(99.5 \%)$ were purchased from Xi'an Polymer Light Technology Co. Ltd., Xi'an, China. N,N-dimethylformamide (DMF, 99.5\%) and Poly( $N$-vinylpyrrolidone) (PVP) with molecular weight (Mw) 130,000 were obtained from Aladdin (Shanghai, China). Another PVP with $\mathrm{Mw} \approx 40,000$ and PVDF pellets ( $\mathrm{Mw} \approx 275,000$ ) were gotten from Sigma-Aldrich (Shanghai, China). $\mathrm{AgNO}_{3}(99.98 \%)$, ethylene glycol and acetone 
were purchased from Beijing Chemical Reagent Co. Ltd., Beijing, China. All the chemicals were used without further purification.

\subsection{Preparation of Perovskite Precursor Solutions}

A series of precursor solutions of PVDF-encapsulated $\mathrm{MAPbBr}_{3-\mathrm{x}} \mathrm{Cl}_{\mathrm{x}}\left(\mathrm{PVDF} \mathrm{MAPbBr}_{3-\mathrm{x}} \mathrm{Cl}_{\mathrm{x}}\right)$ were prepared via a simple stirring process. First, PVDF of $2 \mathrm{~g}$ was poured into $10 \mathrm{~mL}$ DMF and stirred for $1.5 \mathrm{~h}$ at $70{ }^{\circ} \mathrm{C}$ to dissolve it completely. After the PVDF solution slowly cooling down to room temperature, the precursor mixed by $\mathrm{MABr}$ and $\mathrm{PbCl}_{2}$ was added to the PVDF solution during stirring and the precursor solution was obtained after continuous stirring at room temperature for $\sim 2 \mathrm{~h}$. The molar concentrations of $\mathrm{MABr}$ and $\mathrm{PbCl}_{2}$ in the precursor solution were 0.13 and $0.10 \mathrm{M}$, respectively. Similarly, to obtain $\mathrm{MAPbBr}_{3}$ and $\mathrm{MAPbCl}_{3}$, a DMF solution containing both MABr $(0.1 \mathrm{M})$ and $\mathrm{PbBr}_{2}(0.1 \mathrm{M})$, as well as both $\mathrm{MACl}(0.1 \mathrm{M})$ and $\mathrm{PbCl}_{2}(0.1 \mathrm{M})$ was prepared according to the above process, respectively.

\subsection{Synthesis of $A g N P S$}

In preparing Ag NPs, a known quantity of $\mathrm{AgNO}_{3}$ was added to $30 \mathrm{~mL}$ ethylene glycol solution of PVP $(\mathrm{Mw} \approx 40,000)$. The mixture was stirred at $110^{\circ} \mathrm{C}$ for $1 \mathrm{~h}$ in an oil bath. Finally, Ag nanoparticles were collected by centrifugation. It was found that with the benefit of acetone precipitator, the high yield of Ag nanoparticles was obtained by centrifuging the as-prepared solution at 10,000 r/min.

\subsection{Preparation of Ag NPs Electrospinning Solutions}

First, $\mathrm{PVP}(\mathrm{MW} \approx 130,000)$ was dissolved into DMF. In a typical dissolution process, $1 \mathrm{~g}$ PVP was first dissolved in $10 \mathrm{~mL}$ DMF and stirred for $1 \mathrm{~h}$ at the room temperature. Subsequently, the as-produced Ag NPs were added into the PVP solution to prepare a series of electrospinning solutions with Ag NPs concentrations of $0,0.067,0.118,0.236$ and $0.324 \mathrm{M}$, respectively.

\subsection{Preparation of Polymer-Encapsulated Perovskite Electrospinning Membrane with and without Containing} Plasmonic Nanoparticle Nanofibers

Based on the as-prepared solutions, that is, $\mathrm{MAPbBr}_{3-\mathrm{x}} \mathrm{Cl}_{\mathrm{x}}$ precursor solutions or $\mathrm{Ag} \mathrm{NP}$ solutions, we fabricated $\mathrm{MAPbBr}_{3-\mathrm{x}} \mathrm{Cl}_{\mathrm{x}}$ membranes and Ag-enhanced $\mathrm{MAPbBr}_{1.2} \mathrm{Cl}_{1.8}$ membranes by electrospinning, respectively. Herein, needles with diameter of $1.25 \mathrm{~mm}$ were utilized, the distance between the spinning needle tip and the deposited substrate was $10 \mathrm{~cm}$, and the working voltage was set to $19 \mathrm{kV}$. The flow rate of different kinds of solutions was controlled at $0.2 \mathrm{~mL} / \mathrm{h}$. To facilitate the comparison of photoluminescence, we prepared different $\mathrm{MAPbBr}_{3-\mathrm{x}} \mathrm{Cl}_{\mathrm{x}}$ membranes on the same size substrates with the same deposition time $(60 \mathrm{~min})$. Similarly, the membrane of plasmon-enhanced fluorescence of PQDs was fabricated on the substrate of the same size. A layer of PVP-encapsulated Ag NPs (PVP-Ag) which was deposited for 1 min was added at the centrality of the $\mathrm{MAPbBr}_{1.2} \mathrm{Cl}_{1.8}$ membranes and the deposited time for both the lower and the upper $\mathrm{MAPbBr}_{1.2} \mathrm{Cl}_{1.8}$ layers were $30 \mathrm{~min}$.

\subsection{Characterizations}

Photoluminescence spectra were measured via using OmniFluo fluorescence spectrometer (Zolix, Beijing, China). Ultraviolet-visible (UV-vis) absorption spectra were taken on a UV-3600 Plus spectrophotometer (SHIMADZU CO., Ltd., Hong Kong, China). X-ray diffraction (XRD) measurements were measured on a Bruker/D8 FOCUS X-ray diffractometer (Billerica, MA, USA) with a $\mathrm{Cu} \mathrm{K} \alpha$ radiation source (wavelength at $1.5405 \AA$ ). Transmission electron microscope (TEM) and high-resolution TEM (HRTEM) images were recorded with a Technai F20 transmission electron microscope (FEI, Hillsboro, OR, USA). Scanning electron microscopy (SEM) images were taken on a S-4800 SEM microscope (Hitachi, Ltd., Tokyo, Japan). 


\subsection{Theoretical Modeling}

Previously, we calculated optical field distributions of plasmonic nanoparticles (Ag and $\mathrm{Au}$ ) using the finite element method, and derived the plasmon absorption spectra, which can completely reproduce the measured ones $[28,29]$. On the basis of this computing technology, simulation of the optical field distribution of the PVP-Ag nanofiber was performed. Theoretical modeling was based on SEM and TEM measurements of as-prepared samples. The dielectric constants of PVP, PVDF and Ag used here were taken from earlier studies [30-32]. The incident electric field, $E_{\mathrm{y}}=\exp \left(j k_{\mathrm{x}} x\right)$, propagated along the $\mathrm{x}$-axis with polarization in the $\mathrm{y}$-direction. The computational domain was artificially truncated by applying scattering boundary conditions to the upper and lower boundaries, while putting periodic boundary conditions on the left and right boundaries.

\section{Results and Discussion}

\subsection{PVDF-Encapsulated $M A P b B r_{3-x} C l_{x} Q D s$}

A series of uniform large area PVDF-encapsulated $\mathrm{MAPbBr}_{3-\mathrm{x}} \mathrm{Cl}_{\mathrm{x}}\left(\mathrm{PVDF}-\mathrm{MAPbBr}_{3-\mathrm{x}} \mathrm{Cl}_{\mathrm{x}}\right)$ with $x=0,1.8$, and 3 membranes were fabricated by electrospinning. As constituent elements of these membranes, the nanofibers are highly uniform in dimensions and their surfaces are smooth, and, more importantly, PQDs are completely encapsulated inside the nanofibers, thus gaining excellent surface passivation. For example, Figure $1 \mathrm{a}, \mathrm{b}$ show SEM and TEM images of PVDF-MAPbBr ${ }_{1.2} \mathrm{Cl}_{1.8}$ nanofibers. Obviously, almost no PQDs are embedded on the surface of the nanofibers, which makes the surface of the nanofibers smooth (Further support in Figure S1). Notably, the highly efficient passivation of PQDs fabricated by such a simple method is equivalent to that of the perovskite/polymer core/shell nanostructure, whereas this method avoids the tedious processes of synthesizing core/shell nanostructures. The average diameter of as-prepared nanofibers was $68.1 \mathrm{~nm}$ and a histogram of the dimension distribution is shown in Figure S2a.

(a)

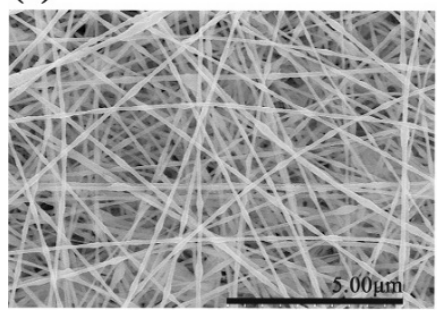

(b)

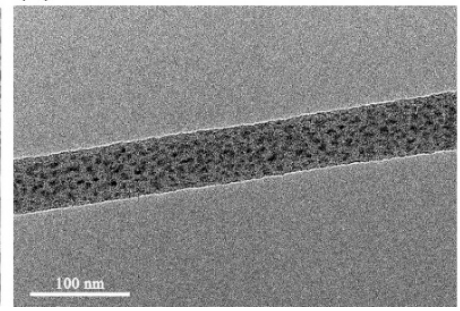

(c)

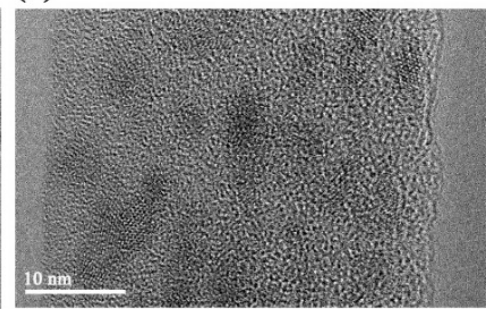

Figure 1. (a) Scanning electron microscopy (SEM) image of polyvinylidene fluoride (PVDF)-MAPbBr ${ }_{1.2} \mathrm{Cl}_{1.8}$ nanofibers. (b) Transmission electron microscope (TEM) image of a single PVDF nanofiber with embedded $\mathrm{MAPbBr}{ }_{1.2} \mathrm{Cl}_{1.8}$. (c) High-resolution TEM (HRTEM) image of $\mathrm{MAPbBr}_{1.2} \mathrm{Cl}_{1.8}$ quantum dots (QDs) in PVDF nanofiber.

A HRTEM image of PVDF-MAPbBr ${ }_{1.2} \mathrm{Cl}_{1.8}$ nanofibers is shown in Figure 1c. Lattice fringes corresponding to $\mathrm{MAPbBr}_{3-\mathrm{x}} \mathrm{Cl}_{\mathrm{x}}(\mathrm{x}=1.8)$ crystals are clearly. The nanocrystals with an average size of $3.41 \mathrm{~nm}$ are monodispersed in the PVDF nanofiber. Figure S2b summarizes the size distribution of the nanocrystals. Intriguingly, the size of PQDs was elongated along the longitudinal direction of the nanofiber. This is considered to be related to the electric field force. In the PVDF solution, perovskite precursor molecules aggregate into nanoclusters. These nanoclusters were elongated by the electric field force during the electrospinning process and subsequently, with the evaporation of solvents, the molecular clusters grow into perovskite nanocrystals in situ.

The fact that the PQDs can retain several nanometers, which is beneficial for luminescence efficiency of QDs, is attributes to the interaction between $\mathrm{MA}^{+}$of $\mathrm{MACl}$ (or $\mathrm{MABr}$ ) and $-\mathrm{CF}_{2}-$ of PVDF [27]. This interaction prevents $\mathrm{MA}^{+}$from aggregating and thus restricts the growth of perovskite molecular clusters. In addition to passivating surface states and restricting the growth of PQDs, 
PVDF can also provide an environment for the long-term stability of PQDs due to its hydrophobicity and solidity. The XRD pattern of the PVDF membrane prepared by electrospinning is shown in Figure 2. An obvious diffraction peak at $20.2^{\circ}$ is corresponded with $\beta$-phase PVDF, indicating a good crystallinity [27].

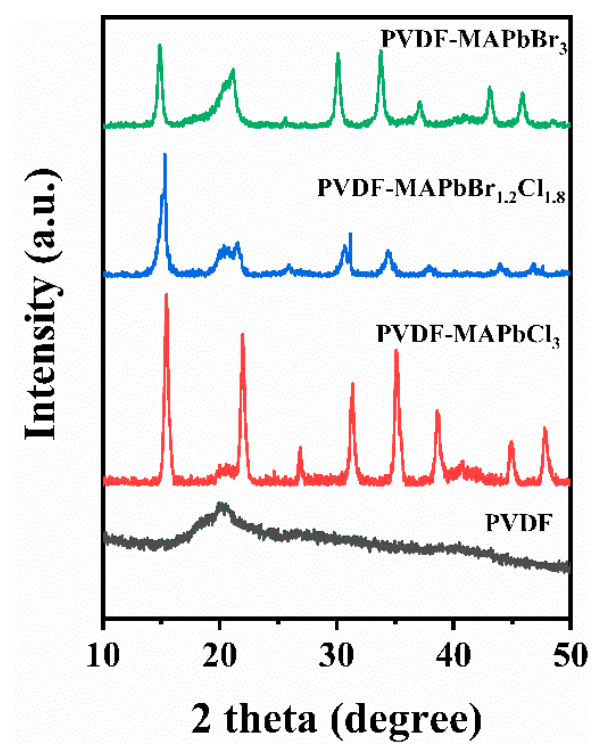

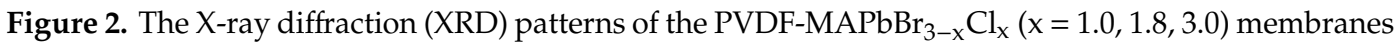
and the PVDF membrane.

\subsection{Effects of Halogens Composition in $\mathrm{MAPbBr}_{3-x} \mathrm{Cl}_{x} \mathrm{QDs}$}

The $\mathrm{MAPbBr}_{3-\mathrm{x}} \mathrm{Cl}_{\mathrm{x}}(x=0,1.8$, or 3$)$ nanocrystals in the PVDF nanofiber can be identified from the XRD patterns (Figure 2). For clarity, the positions of XRD diffraction peaks and their corresponding crystal planes [33] were summarized in Table 1. These diffraction peaks are distinct evidence that the as-synthesized $\mathrm{MAPbBr}_{3-\mathrm{x}} \mathrm{Cl}_{\mathrm{X}}$ is a cubic phase. Intriguingly, by comparing the XRD patterns of $\mathrm{MAPbBr}_{3}, \mathrm{MAPbBr}_{1.2} \mathrm{Cl}_{1.8}$ and $\mathrm{MAPbCl}_{3}$, we found that with increasing the chlorine content in $\mathrm{MAPbBr}_{3-\mathrm{x}} \mathrm{Cl}_{\mathrm{x}}$ nanocrystals, the main diffraction peaks slightly shift towards the larger angle. The main reason is that the radius of chloride ion $(181 \mathrm{pm})$ is smaller than that of bromine ion $(195 \mathrm{pm})$, so the lattice constant of $\mathrm{MAPbBr}_{3-\mathrm{x}} \mathrm{Cl}_{\mathrm{x}}$ nanocrystals tends to decrease with the increase of chlorine content, and according to Bragg diffraction theory, the corresponding diffraction angle increases.

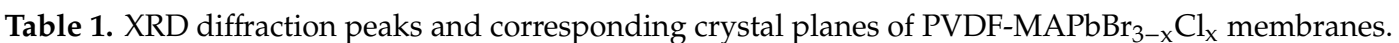

\begin{tabular}{lcccccccc}
\hline \multicolumn{1}{c}{ Samples } & \multicolumn{7}{c}{ Diffraction Peaks and Crystal Planes } \\
\hline $\mathrm{MAPbBr}_{3}$ & $14.82^{\circ}$ & $21.04^{\circ}$ & $25.98^{\circ}$ & $30.11^{\circ}$ & $33.72^{\circ}$ & $37.09^{\circ}$ & $43.09^{\circ}$ & $45.91^{\circ}$ \\
$\mathrm{MAPbBr}_{1.2} \mathrm{Cl}_{1.8}$ & $15.30^{\circ}$ & $21.54^{\circ}$ & $26.34^{\circ}$ & $30.83^{\circ}$ & $34.37^{\circ}$ & $38.06^{\circ}$ & $44.04^{\circ}$ & $46.83^{\circ}$ \\
$\mathrm{MAPbCl}_{3}$ & $15.42^{\circ}$ & $21.97^{\circ}$ & $26.89^{\circ}$ & $31.30^{\circ}$ & $35.11^{\circ}$ & $38.60^{\circ}$ & $44.93^{\circ}$ & $47.85^{\circ}$ \\
Crystal planes & $(001)$ & $(011)$ & $(111)$ & $(002)$ & $(021)$ & $(211)$ & $(022)$ & $(003)$ \\
\hline
\end{tabular}

The effect of halogens composition in PQDs on their spectrum were scrutinized by applying the UV-vis absorption and PL emissions. The normalized light absorption spectra of the PVDF-PQDs membranes are shown in Figure 3a. The strong absorptions start at 405, 455 and $525 \mathrm{~nm}$ for $\mathrm{MAPbCl}_{3}$, $\mathrm{MAPbBr}_{1.2} \mathrm{Cl}_{1.8}$ and $\mathrm{MAPbBr}_{3}$, respectively and are blue-shifted with chlorine replacing bromine, which is associated with bandgap widening upon chlorine doping [13]. According to the Tauc plot (Figure $3 \mathrm{~b}$ ), the bandgaps of $\mathrm{MAPbCl}_{3}, \mathrm{MAPbBr}_{1.2} \mathrm{Cl}_{1.8}$ and $\mathrm{MAPbBr}_{3}$ QDs in the PVDF nanofibers are calculated to be $2.98,2.58$ and $2.28 \mathrm{eV}$, respectively, which was obtained by reading the abscissa value of the intersection of the tangent and the $x$-axis. 
(a)

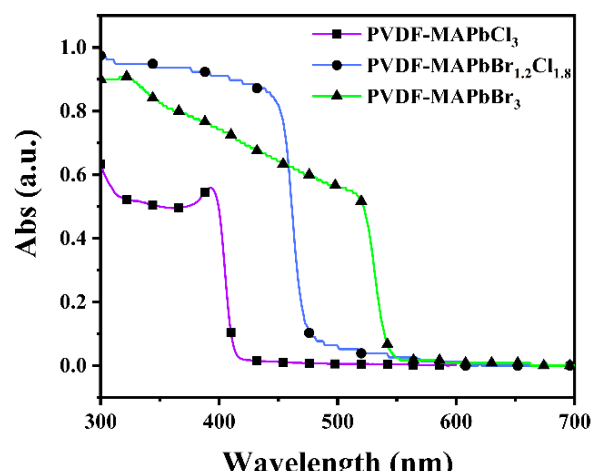

(c)

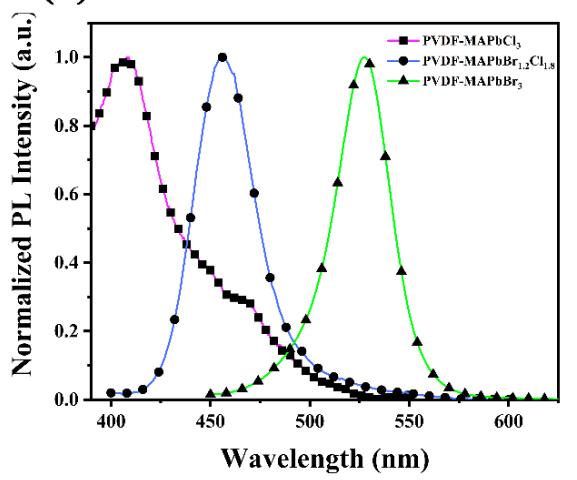

(b)

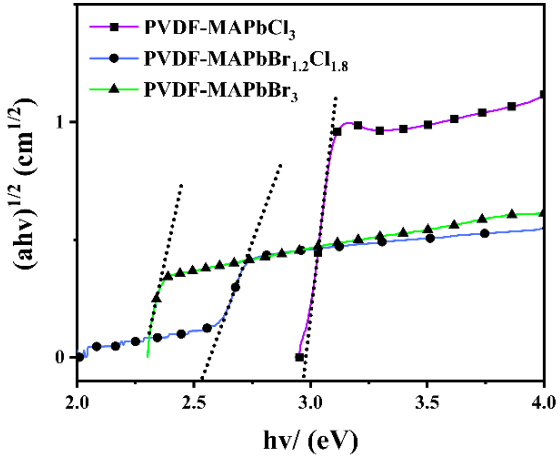

(d)

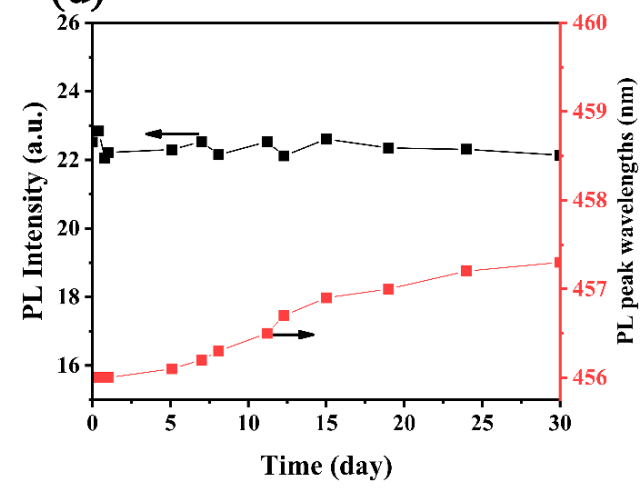

Figure 3. (a) Normalized absorption spectra, (b) Tauc plot and (c) normalized photoluminescence (PL) spectra of PVDF-perovskite quantum dots (PQDs) membranes. (d) Daily record data of the PL peak value and its wavelength position of the PVDF-MAPbBr ${ }_{1.2} \mathrm{Cl}_{1.8}$ membrane which was kept in the air at room temperature.

By adjusting the ratio of $\mathrm{Cl}^{-}$and $\mathrm{Br}^{-}$, a series of PVDF-perovskites membranes of different emission wavelengths were prepared (Figure S3). Especially, Figure 3c compares PL spectra of $\mathrm{MAPbCl}_{3}, \mathrm{MAPbBr}_{1.2} \mathrm{Cl}_{1.8}$ and $\mathrm{MAPbBr}_{3}$ QDs encapsulated in PVDF membranes. Corresponding with the wavelength of the absorption onset, $\mathrm{PL}$ peaks of $\mathrm{MAPbCl}_{3}, \mathrm{MAPbBr}_{1.2} \mathrm{Cl}_{1.8}$ and $\mathrm{MAPbBr}_{3}$ QDs excited by $330 \mathrm{~nm}$ light are at 407, 456, and $527 \mathrm{~nm}$ and their full width at half maximum are of $67.59,31.86$, and $30.47 \mathrm{~nm}$, respectively. To characterize the uniformity of PQDs distribution in the membrane, five points on the membrane were selected to compare their PL intensities (Figure S4a). The PL spectra of the five points were almost coincident, which confirms that the distribution of PQDs is uniform in the membrane (Figure S4b). The comparison of luminous colors of PVDF-MAPbBr ${ }_{1.2} \mathrm{Cl}_{1.8}$ and PVDF-MAPbBr 3 QDs membranes are shown in Figure S5. Furthermore, in comparison to the wavelength of starting absorption, the emission peaks of each sample slightly shifted to the longer wavelength, which is known as the Stokes-shift [34]. Take $\mathrm{MAPbCl}_{3}$ as an example, its PL peak is at $407 \mathrm{~nm}$ wavelength whereas the starting absorption wavelength is at $405 \mathrm{~nm}$. Because the well-crystallized PVDF is solid and compact, it is more suitable for the protection of PQDs. The PL peak value and its wavelength position of a random sample of $\mathrm{PVDF}-\mathrm{MAPbBr}{ }_{1.2} \mathrm{Cl}_{1.8}$ were monitored for 30 days under the same measuring conditions, as shown in Figure 3d. Notably, the PL attenuation is less than $1 \%$, while the wavelength shift is within $1 \mathrm{~nm}$ due to the slight growth of the PQD gains, which indicates an excellent stability of the PVDF-PQDs.

\subsection{Plasmon-Enhanced Fluorescence}

In comparison to $\mathrm{MAPbBr}_{3}$ QDs, the luminous efficiency of $\mathrm{MAPbBr}_{3-\mathrm{x}} \mathrm{Cl}_{\mathrm{x}}$ QDs has been hovering around very low values due to the increase in the undesirable effects of defect states. To improve the luminous efficiency, we explored plasmon-enhanced fluorescence of $\mathrm{MAPbBr}_{3-\mathrm{x}} \mathrm{Cl}_{\mathrm{x}}$ by introducing 
PVP-Ag nanofibers into PQD nanofibers membranes. For PVP-Ag nanofibers, the Ag NPs were encapsulated by PVP which acts as a good stabilizer. Two sp orbitals of the $\mathrm{Ag}^{+}$ions could be occupied by the donated lone pair electrons of both oxygen and nitrogen atoms in PVP monomers, which effectively stabilizes $\mathrm{Ag}^{+}$ions and decreases their chemical potential [35]. Figure $4 \mathrm{a}$ and $\mathrm{b}$ display HRTEM images of PVP-Ag nanofiber and Ag NPs. Clearly, the Ag NPs are well-dispersed in a nanofiber without aggregation, and these particles are highly spherical with an average size $13 \mathrm{~nm}$. The absorbance spectrum of Ag NPs was measured in DMF solution, which shows a strong absorption peak at $470 \mathrm{~nm}$. $\mathrm{MAPbBr}_{1.2} \mathrm{Cl}_{1.8}$ nanocrystals were selected for plasmon-enhanced experiments owing to its PL spectrum coinciding well with the plasmonic resonance band of Ag NPs (shown in Figure 4c), which promisingly produced a high fluorescence enhancement $[23,24]$. PVP-Ag nanofibers and PQD nanofibers were deposited on a substrate by layer-by-layer electrospinning to form a composite membrane, where Ag nanofibers of each layer interweaved with PQD nanofibers of the adjacent layer.

\section{(a)}

(b)
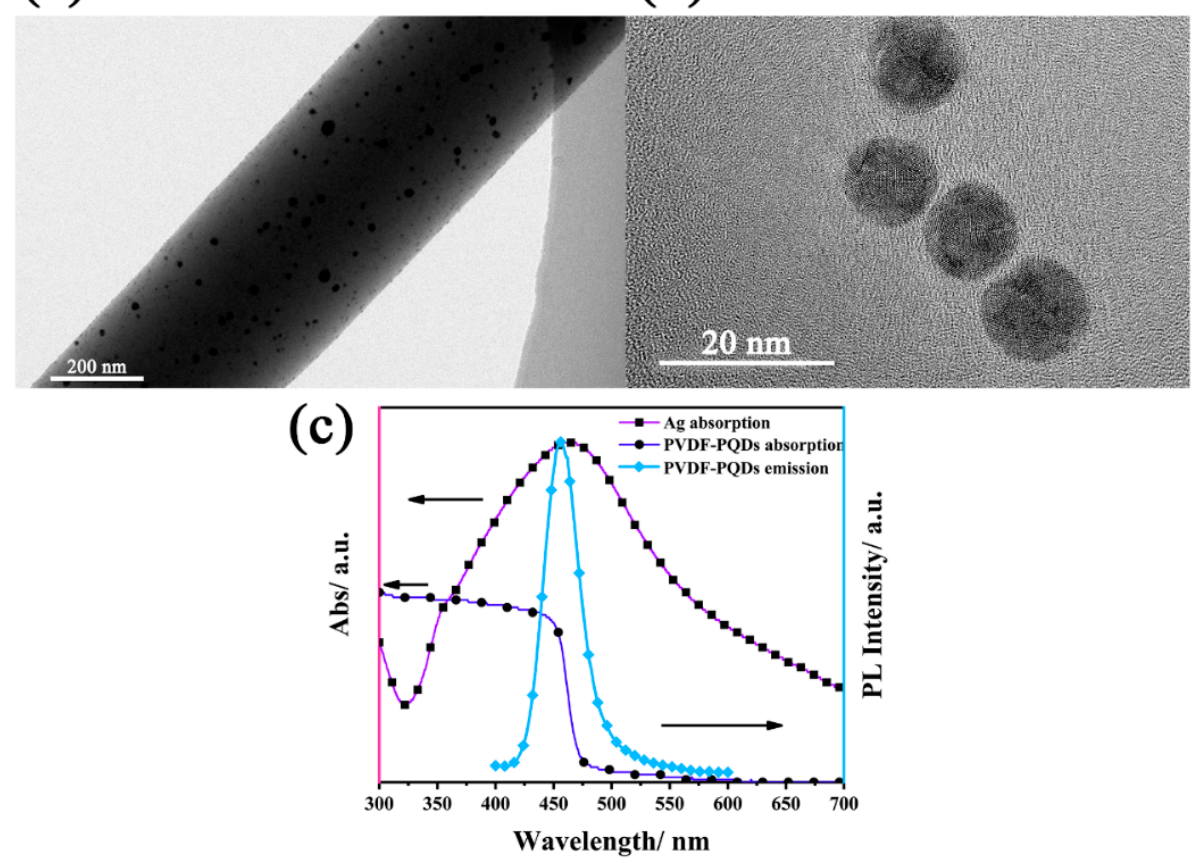

Figure 4. HRTEM images of the (a) PVP-Ag nanofiber and (b) silver nanoparticles (Ag NPs); (c) Absorbance spectra of $\mathrm{Ag} \mathrm{NPs}$ and PVDF-MAPbBr ${ }_{1.2} \mathrm{Cl}_{1.8}$, and PL spectrum of PVDF-MAPbBr ${ }_{1.2} \mathrm{Cl}_{1.8}$.

We defined $\mathrm{MAPbBr}_{1.2} \mathrm{Cl}_{1.8}$-layer/Ag-layer/ $\mathrm{MAPbBr}{ }_{1.2} \mathrm{Cl}_{1.8}$-layer as an analytical periodic unit as shown in Figure $5 \mathrm{a}$. Figure $5 \mathrm{~b}$ compares the emission spectra of periodic units with and without an Ag-layer. Clearly, in comparison with the $\mathrm{MAPbBr}_{1.2} \mathrm{Cl}_{1.8}$ membrane, $\mathrm{Ag}$ nanofibers located in the $\mathrm{MAPbBr}_{1.2} \mathrm{Cl}_{1.8}$ membrane can significantly enhance the PL intensity. The enhancement effect is affected by the concentration of Ag NPs in PVP nanofibers. The PL signal increases as more and more $\mathrm{Ag}$ NPs have been introduced in nanofibers up to a certain concentration. The maximum fluorescence intensity was enhanced to be $\sim 4.8$ folds of initial fluorescence intensity after the additive amount of Ag NPs to the optimized concentration 0.236 M. However, since Ag NPs increased beyond the optimal concentration, fluorescence intensity decreased, which is aligned with previous results [23,24]. It is believed that in addition to enhancing fluorescence, Ag NPs also act as absorbers and scatters of the fluorescence of $\mathrm{MAPbBr}_{1.2} \mathrm{Cl}_{1.8}$ QDs, and with the increase concentration of nanoparticles, the fluorescence loss becomes dominant. A different explanation is proposed later in this paper based on the investigation of optical field distribution of Ag nanofibers. 
(a)

(b)

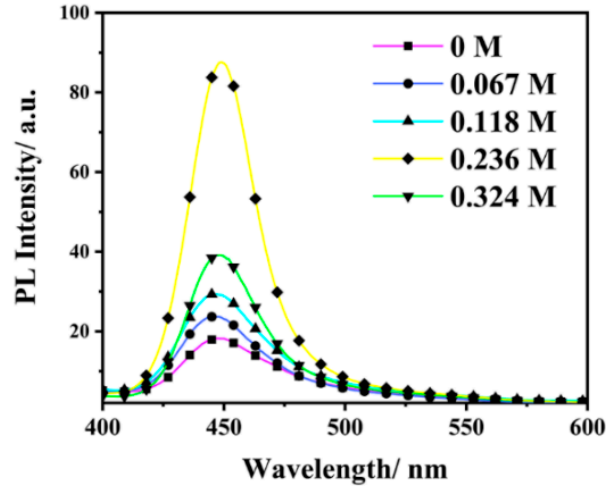

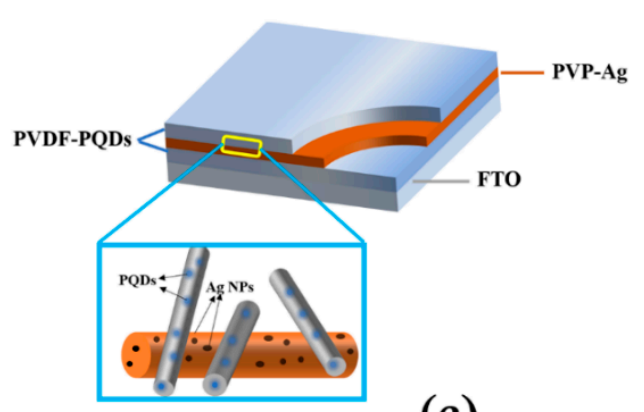

(c)

Figure 5. (a) The schematic diagram of the multilayer membrane structure. The insert is enlarged view of PVP-Ag and PVDF-PQD nanofibers on the interface between PVP-Ag and PQD layers (b) PL spectra of the composite membranes with a layer of PVP-Ag nanofibers sandwiched in the $\mathrm{MAPbBr}_{1.2} \mathrm{Cl}_{1.8}$ membranes, and the concentrations of $\mathrm{Ag}$ NPs varying from 0 to $0.324 \mathrm{M}$. (c) Normalized PL spectra of PVDF-MAPbBr ${ }_{1.2} \mathrm{Cl}_{1.8}$ membranes, the sandwich structural with a PVP film between the PVDF-MAPbBr ${ }_{1.2} \mathrm{Cl}_{1.8}$ films and the sandwich structural with PVP-Ag nanofiber film between the PVDF-MAPbBr ${ }_{1.2} \mathrm{Cl}_{1.8}$ films.

Moreover, in comparison with the PVDF-MAPbBr${ }_{1.2} \mathrm{Cl}_{1.8}$ single layer film, the maximum emission peak of the multi-layer film shows the $\sim 8 \mathrm{~nm}$ blue-shift as shown in Figure $5 \mathrm{c}$. This blue shift is caused by the introduction of PVP rather than Ag NPs. To prove this, we introduced a layer of pure PVP-nanofiber film at the middle of the PVDF-MAPbBr ${ }_{1.2} \mathrm{Cl}_{1.8}$ membrane. Obviously, the PL peak shifts $\sim 8 \mathrm{~nm}$ to the short wavelength. However, no blue or red shift of the PL peak was observed upon adding Ag NPs into the PVP-nanofiber.

\subsection{Theoretical Analysis of the Plasmon-Enhanced Fluorescence}

The plasmon-enhanced luminescence is usually caused by the local electric field intensification close to the metallic surface. For too-short distances between the emitter and the metal surface, however, non-radiative transitions are usually dominant, thereby quenching the radiative emission. The quenching is usually caused by energy losses in the metal, such as interband transitions [36]. In the proposed structure (Figure 5a), the polymers (PVDF and PVP) that are highly transparent in the emission range of $\mathrm{MAPbBr}_{3-x} \mathrm{Cl}_{x}$ QDs separate the emitters from Ag NPs at an appropriate distance, and therefore eliminate the fluorescence quenching. According to TEM measurements of PVDF-PQDs nanofibers and PVP-Ag nanofibers, the average nearest distance between PQDs and Ag NPs can be deduced to be $7 \mathrm{~nm}$, which is within the near-field enhancement of plasmonic nanoparticles [26,36]. In addition to the near-field, it is reported that the far-field of plasmonic nanoparticles also contributes to the fluorescence enhancement [26]. In order to gain insight into the fluorescence enhancement ability of PVP-Ag nanofibers, we simulated the optical field distribution of the PVP-Ag nanofiber irradiated by the $330 \mathrm{~nm}$ light that excites the fluorescence of $\mathrm{MAPbBr}_{1.2} \mathrm{Cl}_{1.8}$ QDs. On the basis of SEM and TEM 
observation on as-prepared NPs, a calculation model representing PVP-Ag nanofiber containing a certain Ag NPs concentration in the PVP membrane is established. The geometric design of Ag NPs and polymer nanofibers was determined by the average measured values of as-prepared samples. In particular, Ag NPs with average size of $13 \mathrm{~nm}$ are distributed in a PVP nanofiber at the particle density of 30 per nanometer in the simulated model, and the diameter ratio of PVP nanofiber to PVDF nanofiber is $\sim 5$. Light scattering by PQDs with only a few nanometers is negligible, hardly effecting the optical field distribution of the PVP-Ag nanofiber [37]. Therefore, in order to highlight the main factors, PQDs are not included in the PVDF-nanofiber computational model. Figure $6 \mathrm{c}$ shows the optical field distribution of PVP-Ag nanofiber. Surprisingly, in comparison with the incident optical field, the strong optical field distributes within the larger region around the Ag nanofiber, which is consistent with the reported far-field light enhancement of plasmonic nanoparticles [26]. The optical field intensity at just several nanometers away from Ag NPs can be enhanced to $10^{5}$ times of the background optical field. This is undoubtedly one of the main reasons for the fluorescence enhancement of $\mathrm{MAPbBr}_{1.2} \mathrm{Cl}_{1.8} \mathrm{QDs}$ by adding $\mathrm{Ag}$ nanofibers in the $\mathrm{MAPbBr}_{1.2} \mathrm{Cl}_{1.8}$ QD membrane.
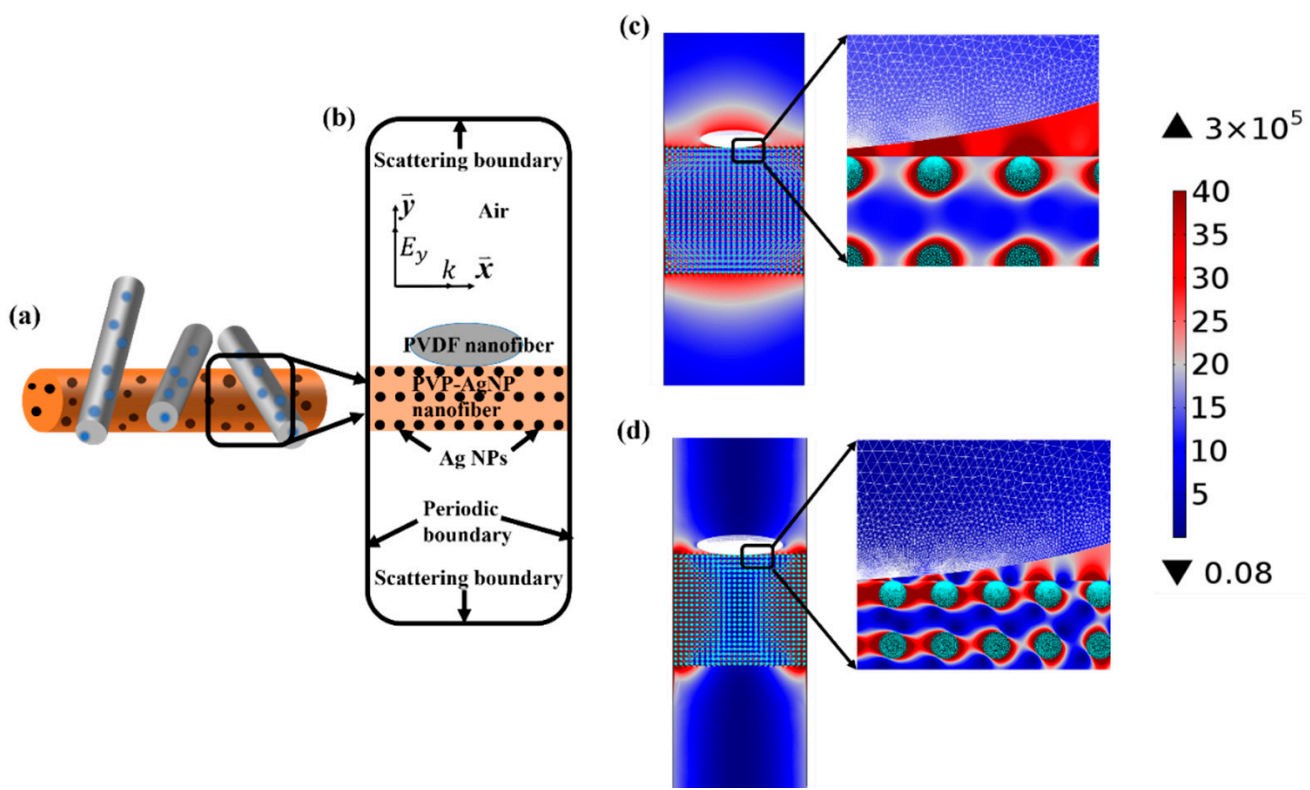

Figure 6. (a) The schematic diagram of the intertexture of PVP-Ag nanofibers and PQD nanofibers on the interface between PVP-Ag and PQD layers; (b) a cross-section of the computational geometry. The calculation dimension is truncated by scattering boundary conditions and periodic boundary conditions. The incident optical wave is polarized along $y$-axis, traveling along $x$-axis. $E_{y}$ and $k$ are the incident electric field and wavevector, respectively; (c) and (d) Plots of relative optical field intensity $E^{2}=\left(E \cdot E^{*}\right) /\left|E_{\text {inc }}\right|^{2}$ corresponding to 0.236 and $0.324 \mathrm{M}$ of Ag NPs concentration in PVP-Ag nanofibers, respectively, where $\left(E \cdot E^{*}\right)$ is the amplitude of the total optical field intensity and $\left|E_{i n c}\right|^{2}=1 \mathrm{~J} / \mathrm{nm}^{2}$ is the amplitude of the incident optical field intensity. The inserts in both (c) and (d) are enlarged view of the distribution of optical field intensity at the intersection of both PVP-Ag and PQD nanofibers.

It is noteworthy that in the model simulated above, the density of Ag NPs in PVP nanofiber is 30/nm, corresponding to the average measured density of Ag NPs in as-prepared PVP-Ag nanofibers obtained by using the optimized concentration $0.236 \mathrm{M}$ of Ag NPs. When the concentration of Ag NPs increased to $0.324 \mathrm{M}$, the average measured density of Ag NPs in as-prepared PVP-Ag nanofibers is 40/nm. On the basis of this density, the optical field around Ag nanofiber was simulated again and the results are shown in Figure 6d. Intriguingly, the optical field of Ag NPs shows strong locality and most of the light energy is concentrated between adjacent Ag NPs due to plasmon resonance coupling. However, the extended range of the enhanced light field around Ag NPs decreases, indicating that the 
enhanced range of fluorescence reduces accordingly. Therefore, we believe this is the main reason for fluorescence intensity decreases upon increasing Ag NPs beyond the optimal concentration.

\section{Conclusions}

In summary, we have developed a cost-efficient high-throughput strategy for fabricating stable PVDF-PQDs membranes using the electrospinning method. With PVDF completely encapsulating, PQDs were excellently surface-passivated and well insulated from water and oxygen. Moreover, the molecule group $-\mathrm{CF}_{2}-$ of PVDF prevents $\mathrm{MA}^{+}$of PQDs from aggregating, thus enabling PQDs to retain several nanometers, which is beneficial for luminescence efficiency. A series of PVDF-MAPbBr $r_{3-x} \mathrm{Cl}_{x}$ membranes were fabricated. By controlling the ratio of $\mathrm{Cl}^{-}$to $\mathrm{Br}^{-}$of $\mathrm{MAPbBr}_{3-\mathrm{x}} \mathrm{Cl}_{\mathrm{x}}$, the position of the PL peak varies from 402 to $527 \mathrm{~nm}$, so that the color of the emitting light can be tuned flexibly. To improve luminous efficiency, we successfully introduced a layer of PVP-Ag nanofibers into the PVDF-PQDs membrane via layer-by-layer electrospinning and this well-considered structure produced 4.8 folds fluorescence enhancement of the PQDs. The enhancement effect of Ag nanofibers was scrutinized by simulating the optical field distribution of PVP-Ag nanofiber. Our strategy is robust as it can be easily extended to fabricate polymer-encapsulated nanoparticle membranes of diversified materials with engineered properties for applications in LEDs, lasers, light-emitting transistors and photovoltaic devices.

Supplementary Materials: The following are available online at http://www.mdpi.com/2079-4991/9/5/770/s1, Figure S1: TEM and HRTEM images of PVDF-MAPbBr ${ }_{1.2} \mathrm{Cl}_{1.8}$ nanofibers indicating that almost all PQDs are encapsulated inside the PVDF fibers, Figure S2: (a) The diameter statistics of the PVDF-MAPbBr ${ }_{1.2} \mathrm{Cl}_{1.8}$ fibers and (b) the size statistics of the MAPbBr ${ }_{1.2} \mathrm{Cl}_{1.8}$ QDs, Figure S3: Normalized PL spectra of PVDF-perovskites with different the ratio of $\mathrm{Cl}^{-}$and $\mathrm{Br}^{-}$, Figure S4: (a) Schematic diagram of five points on the PVDF-PQDs membrane; (b) The PL spectra of five points selected on the membrane and the inset shows the overlap of the PL spectra of these five points. Figure S5: The optical images of (a) PVDF-MAPbBr ${ }_{1.2} \mathrm{Cl}_{1.8}$ and (b) PVDF-MAPbBr $\mathrm{Pr}_{3}$ films on FTO substrates under ultraviolet irradiation at $\sim 365 \mathrm{~nm}$ wavelength.

Author Contributions: K.G. performed the experiments and the data analysis; S.H. synthesized Ag nanoparticles; H.P. performed the data analysis; D.Y. performed the data analysis and wrote the paper. All authors contributed to the general discussion.

Funding: This research was funded by the National Science Foundation of China, grant numbers 61875234 and 61775245, and the National Study Fund Committee of China, grant number 2016-QT-049.

Conflicts of Interest: The authors declare no conflict of interest.

\section{References}

1. Yang, Y.; You, J.; Meng, L. Efficient and Stable Perovskite Solar Cells with All Solution Processed Metal Oxide Transporting Layers. U.S. Patent 15/553,483, 1 February 2018.

2. Chang, S.; Bai, Z.; Zhong, H. In situ fabricated perovskite nanocrystals: A revolution in optical materials. Adv. Opt. Mater. 2018, 6, 1800380. [CrossRef]

3. Imran, M.; Caligiuri, V.; Wang, M.; Goldoni, L.; Prato, M.; Krahne, R.; De, T.L.; Manna, L. Benzoyl Halides as Alternative Precursors for the Colloidal Synthesis of Lead Based Halide Perovskite Nanocrystals. J. Am. Chem. Soc. 2018, 140, 2656-2664. [CrossRef]

4. Brenner, T.M.; Egger, D.A.; Kronik, L.; Hodes, G.; Cahen, D. Hybrid organic-Inorganic perovskites: Low-cost semiconductors with intriguing charge-transport properties. Nat. Rev. Mater. 2016, 1, 15007. [CrossRef]

5. Pan, J.; Quan, L.N.; Zhao, Y.; Peng, W.; Murali, B.; Sarmah, S.P.; Yuan, M.; Sinatra, L.; Alyami, N.M.; Liu, J. Highly Efficient Perovskite-Quantum-Dot Light-Emitting Diodes by Surface Engineering. Adv. Mater. 2016, 28, 8718-8725. [CrossRef]

6. Ling, Y.; Yuan, Z.; Tian, Y.; Wang, X.; Wang, J.C.; Xin, Y.; Hanson, K.; Ma, B.; Gao, H. Bright Light-Emitting Diodes Based on Organometal Halide Perovskite Nanoplatelets. Adv. Mater. 2016, 28, 305-311. [CrossRef]

7. Wang, C.; Chesman, A.S.; Jasieniak, J.J. Stabilizing the cubic perovskite phase of $\mathrm{CsPbI}_{3}$ nanocrystals by using an alkyl phosphinic acid. Chem. Commun. 2017, 53, 232-235. [CrossRef] [PubMed]

8. Wei, Y.; Cheng, Z.; Lin, J. An overview on enhancing the stability of lead halide perovskite quantum dots and their applications in phosphor-converted LEDs. Chem. Soc. Rev. 2019, 48, 310-350. [CrossRef] [PubMed] 
9. Saliba, M.; Wood, S.M.; Patel, J.B.; Nayak, P.K.; Huang, J.; Alexander-Webber, J.A.; Wenger, B.; Stranks, S.D.; Hörantner, M.T.; Wang, J.T. Structured Organic-Inorganic Perovskite toward a Distributed Feedback Laser. Adv. Mater. 2016, 28, 923-929. [CrossRef] [PubMed]

10. Zou, S.; Liu, Y.; Li, J.; Liu, C.; Feng, R.; Jiang, F.; Li, Y.; Song, J.; Zeng, H.; Hong, M. Stabilizing Cesium Lead Halide Perovskite Lattice through Mn (II)-Substitution for Air-Stable Light-Emitting Diodes. J. Am. Chem. Soc. 2017, 139, 11443. [CrossRef] [PubMed]

11. Huang, H.; Zhao, F.; Liu, L.; Zhang, F.; Wu, X.G.; Shi, L.; Zou, B.; Pei, Q.; Zhong, H. Emulsion Synthesis of Size-Tunable $\mathrm{CH}_{3} \mathrm{NH}_{3} \mathrm{PbBr}_{3}$ Quantum Dots: An Alternative Route toward Efficient Light-Emitting Diodes. ACS Appl. Mater. Interfaces 2015, 7, 28128. [CrossRef] [PubMed]

12. Bai, S.; Yuan, Z.; Feng, G. Colloidal metal halide perovskite nanocrystals: Synthesis, characterization, and applications. J. Mater. Chem. C 2016, 4, 3898-3904. [CrossRef]

13. Meng, Z.; Hua, Y.; Miaoqiang, L.; Qiong, W.; Jung-Ho, Y.; Lianzhou, W. Composition-dependent photoluminescence intensity and prolonged recombination lifetime of perovskite $\mathrm{CH}_{3} \mathrm{NH}_{3} \mathrm{PbBr}_{3-x} \mathrm{Cl}_{x}$ films. Chem. Commun. 2014, 50, 11727-11730.

14. Leng, M.; Yang, Y.; Chen, Z.; Gao, W.; Zhang, J.; Niu, G.; Li, D.; Song, H.; Zhang, J.; Jin, S. Surface passivation of bismuth-based perovskite variant quantum dots to achieve efficient blue emission. Nano Lett. 2018, 18, 6076-6083. [CrossRef] [PubMed]

15. Wang, Y.; He, J.; Chen, H.; Chen, J.; Zhu, R.; Ma, P.; Towers, A.; Lin, Y.; Gesquiere, A.J.; Wu, S.T. Ultrastable, Highly Luminescent Organic-Inorganic Perovskite-Polymer Composite Films. Adv. Mater. 2016, 28, 10710-10717. [CrossRef] [PubMed]

16. Hengyue, L.I.; Gong, C.; Huang, K.; Yang, J. A Review on the Fabrication of Perovskite Solar Cells via Printing Techniques. Mater. Rev. 2018, 32, 1385-1400.

17. Murphy, J.P.; Andriolo, J.M.; Sutton, N.J.; Brockway, M.C.; Skinner, J.L. Coaxial hybrid perovskite fibers: Synthesis and encapsulation in situ via electrospinning. J. Vac. Sci. Technol. B Nanotechnol. Microelectron. Mater. Process. Meas. Phenom. 2017, 35, 06G402. [CrossRef]

18. Chen, D.; Zhu, Y. Electrospun Perovskite Nanofibers. Nanoscale Res. Lett. 2017, 12, 114. [CrossRef] [PubMed]

19. Chao, L.-M.; Tai, T.-Y.; Chen, Y.-Y.; Lin, P.-Y.; Fu, Y.-S. Fabrication of $\mathrm{CH}_{3} \mathrm{NH}_{3} \mathrm{PbI}_{3} / \mathrm{PVP}$ Composite Fibers via Electrospinning and Deposition. Materials 2015, 8, 5467-5478. [CrossRef]

20. Tsai, P.C.; Chen, J.Y.; Ercan, E.; Chueh, C.C.; Tung, S.H.; Chen, W.C. Uniform Luminous Perovskite Nanofibers with Color-Tunability and Improved Stability Prepared by One-Step Core/Shell Electrospinning. Small 2018, 14, 1704379. [CrossRef]

21. Lin, C.C.; Jiang, D.H.; Kuo, C.C.; Cho, C.J.; Tsai, Y.H.; Satoh, T.; Su, C. Water-Resistant Efficient Stretchable Perovskite-Embedded Fiber Membranes for Light-Emitting Diodes. ACS Appl. Mater. Interfaces 2018, 10, 2210. [CrossRef]

22. Aslan, K.; Wu, M.; Lakowicz, J.R.; Geddes, C.D. Fluorescent core-shell Ag@SiO 2 nanocomposites for metal-enhanced fluorescence and single nanoparticle sensing platforms. J. Am. Chem. Soc. 2007, 129, 1524. [CrossRef] [PubMed]

23. Dadı, S.; Altıntas, Y.; Beskazak, E.; Mutlugun, E. Plasmon Enhanced Emission of Perovskite Quantum Dot Films. MRS Adv. 2018, 3, 733-739. [CrossRef]

24. Pompa, P.P.; Martiradonna, L.; Torre, A.D.; Sala, F.D.; Manna, L.; De Vittorio, M.; Calabi, F.; Cingolani, R.; Rinaldi, R. Metal-enhanced fluorescence of colloidal nanocrystals with nanoscale control. Nat. Nanotechnol. 2006, 1, 126-130. [CrossRef] [PubMed]

25. Tovmachenko, O.; Graf, C.; Heuvel, D.J.V.D.; Blaaderen, A.V.; Gerritsen, H.C. Fluorescence Enhancement by Metal-Core/Silica-Shell Nanoparticles. Adv. Mater. 2010, 18, 91-95. [CrossRef]

26. Wu, X.; Li, Y.; Wu, L.; Fu, B.; Liu, G.; Zhang, D.; Zhao, J.; Chen, P.; Liu, L. Enhancing perovskite film fluorescence by simultaneous near- and far-field effects of gold nanoparticles. RSC Adv. 2017, 7, 35752-35756. [CrossRef]

27. Zhou, Q.; Bai, Z.; Lu, W.G.; Wang, Y.; Zou, B.; Zhong, H. In Situ Fabrication of Halide Perovskite Nanocrystal-Embedded Polymer Composite Films with Enhanced Photoluminescence for Display Backlights. Adv. Mater. 2016, 28, 9163-9168. [CrossRef] [PubMed]

28. Yang, D.; Pang, X.; He, Y.; Wang, Y.; Chen, G.; Wang, W.; Lin, Z. Precisely size-tunable magnetic/plasmonic core/shell nanoparticles with controlled optical properties. Angew. Chem. 2015, 127, 12259-12264. [CrossRef] 
29. Yang, D.; Chen, Y.; Peng, H.S.; Chen, G.; Lin, Z. An integrated experimental and theoretical study on optical properties of uniform hairy noble metal nanoparticles. Nanoscale 2018, 10, 22750-22757. [CrossRef]

30. Alias, A.; Kudin, T.; Zabidi, Z.; Harun, M.K.; Ali, A.M.M.; Yahya, M.F. In Refractive index dispersion and optical dielectric properties of poly(N-carbazole)/poly(vinylpyrrolidone) blends. Adv. Mater. Res. 2013, 652-654, 532-536. [CrossRef]

31. Bai, M.; Sorokin, A.; Thompson, D.W.; Poulsen, M.; Ducharme, S.; Herzinger, C.; Palto, S.; Fridkin, V.; Yudin, S.; Savchenko, V. Determination of the optical dispersion in ferroelectric vinylidene fluoride (70\%)/trifluoroethylene (30\%) copolymer Langmuir-Blodgett films. J. Appl. Phys. 2004, 95, 3372-3377. [CrossRef]

32. Johnson, P.B.; Christy, R.-W. Optical constants of the noble metals. Phys. Rev. B 1972, 6, 4370. [CrossRef]

33. Baikie, T.; Barrow, N.S.; Fang, Y.; Keenan, P.J.; Slater, P.R.; Piltz, R.O.; Gutmann, M.; Mhaisalkar, S.G.; White, T.J. A combined single crystal neutron/X-ray diffraction and solid-state nuclear magnetic resonance study of the hybrid perovskites $\mathrm{CH}_{3} \mathrm{NH}_{3} \mathrm{PbX}_{3}(\mathrm{X}=\mathrm{I}, \mathrm{Br}$ and $\mathrm{Cl})$. J. Mater. Chem. A 2015, 3, 9298-9307. [CrossRef]

34. Brennan, M.C.; Herr, J.E.; Nguyen-Beck, T.S.; Zinna, J.; Draguta, S.; Rouvimov, S.; Parkhill, J.; Kuno, M. Origin of the size-dependent stokes shift in $\mathrm{CsPbBr}_{3}$ perovskite nanocrystals. J. Am. Chem. Soc. 2017, 139, 12201-12208. [CrossRef] [PubMed]

35. Jin, W.J.; Lee, H.K.; Jeong, E.H.; Park, W.H.; Ji, H.Y. Preparation of Polymer Nanofibers Containing Silver Nanoparticles by Using Poly(N-vinylpyrrolidone). Macromol. Rapid Commun. 2010, 26, 1903-1907. [CrossRef]

36. Guidelli, E.J.; Ramos, A.P.; Baffa, O. Enhancing and quenching luminescence with gold nanoparticle films: The influence of substrate on the luminescent properties. Nanotechnology 2015, 27, 015503. [CrossRef]

37. Davletshin, Y.R.; Lombardi, A.; Cardinal, M.F.; Juvé, V.; Crut, A.; Maioli, P.; Liz-Marzan, L.M.; Vallée, F.; Fatti, N.D.; Kumaradas, J.C. A quantitative study of the environmental effects on the optical response of gold nanorods. ACS Nano 2012, 6, 8183-8193. [CrossRef] 